\section{The Mechanism of Formation of the Latent Photographic Image.}

A KNOWLEDGE of the process whereby the latent image is produced on the exposure of a photographic emulsion to light is of primary importance to those engaged in fundamental photographic research. For some years past this problem has been under investigation in the laboratories of the British Photographic Research Association, and considerable progress has recently been made in our knowledge of the primary light action.

As a first step, our method has been to try to identify the photographic mechanism with some characteristic of the silver halides which can be studied by purely physical methods, in the absence of such disturbing factors as gelatin, etc.

It has been known for a long time that the silver halides possess both photo-electric and photoconductivity properties. By the former is meant the complete liberation of electrons from the salt under light action, and by the latter the freeing of electrons internally, resulting in a change of conductivity on illumination (sometimes called the internal photoelectric effect). It seemed possible to us that the mechanism responsible for one of these effects might be identified with that which produces the latent photographic image, and consequently we started more than two years ago a series of experiments to investigate thoroughly the two effects in relation to the silver halides, and especially to silver bromide.

The results of a long series of experiments which were published (Phil. Mag., 3, 482; 1927) indicated that the photographic mechanism was not photoelectric in the sense of its being a complete liberation of electrons from the crystal of silver bromide. The evidence in support of this conclusion is that photographic action takes place in an emulsion made with silver bromide at wave-lengths very much longer than the longest which will produce any photo-electric emission from that salt.

A detailed investigation of the second, i.e. photoconductivity effect, has now been in progress for more than a year, and the results already obtained seem to be sufficiently striking to warrant publication in the form of a preliminary note.

A study of the literature indicated that in many ways a parallelism does exist between photoconductivity and photographic effects. For example, the spectral regions to which emulsions are sensitive photographically are the same on the long wave. length side as those to which the corresponding pure halides show photo-conductivity effects. Take the most important case-that of silver bromide. The photographic spectral sensitivity of a slow silver bromide emulsion increases very rapidly towards the blue from practically nothing at about $\lambda 5000$. The very trustworthy experiments of Coblentz (U.S. Bureau of Standards, Scientific Paper No. 256) on the photoconductivity of silver bromide showed that this effect also commences in the same spectral region as the photographic effect and increases also towards the blue. To this extent, therefore, the two effects are parallel. Coblentz's results, however, showed a very serious departure from the photographic case in that as the wave-length decreased from $\lambda 5000$, the photo-conductivity effect (for equal energy) after rising rapidly to a maximum, fell practically to zero at about $\lambda 4200$. This, at first sight, seemed to indicate that the mechanisms producing the two effects are different, because it is well known that photographic action occurs at wave-lengths far shorter than $\dot{\lambda} 4200$.

In the case of such thickly coated plates as are commonly used in photography, there is an apparent decrease of sensitivity as we pass from the violet to the ultra-violet. This effect has, however, been shown (Phil. Mag., 49, 1104; 1925) to be due, not to a real decrease in sensitivity of the grains of silver bromide, but to secondary effects involving the thicknoss of the sensitive film. Fxperiments with thinly coated plates demonstrated (Trans. Faraday Soc., 19, 290; 1923; Phil. Mag., 48, 947; 1924) that the photographic sensitivity of silver bromide actually increases on passing from the violet to the ultra-violet. It was thought that the sharp decrease of photo-conductivity in the violet and the apparent absence of the effect in the ultra-violet reported by Coblentz might also have been due to a "thickness" effect in the specimens used by him and was not an inhercnt characteristic of the silver bromido.

Since the light absorption by silver bromide increases extremely rapidly with decreasing wavelength from the blue to the ultra-violet, the former light penetrates much farther than the latter into the silver bromide layer. Since, further, the electrical conductivity depends on the whole thickness of the layer, it was suspected that the relative photoconductivity effects at different wave-lengths depends on this thickness, just as we have shown the photographic effect so to depend. Experiments were therefore undertaken to measure the photo-conductivity effects at the three wave-lengths $\lambda 4385$ (blue), $\lambda 4060$ (violet), and $\lambda 3650$ (ultra-violet), and to see whether their relative values varied with the thickness of the silver bromide specimen employed.

Preliminary experiments have completely verified the predictions, and have shown that while with a fairly thick specimen (about $0.7 \mathrm{~mm}$.) the relative order of effects is blue $>$ violet $>$ ultra-violet, yet as the thickness of the specimen is decreased, so the violet and ultra-violet increase relatively to the blue, the order given above becoming completely reversed with a sufficiently thin specimen. The thinnest yet measured is about $0.07 \mathrm{~mm}$. thick, and even for this the effect of a given amount of energy is about twice as great at $\lambda 3650$ as at $\lambda 4358$. Thus what seems to have been the greatest difficulty in the way of demonstrating the identity of the photographic mechanism and that which produces a change in electrical conductivity on illumination, has been removed.

There is considerable experimental evidence that the mechanism of the photo-conductivity effect observed with many crystalline metallic halides involves the loosening of electrons from the halide ions of the lattice. The present work therefore adds considerable weight to the hypothesis that latent image formation involves the transfer of valency electrons from bromide ions to silver ions, resulting in the formation of metallic silver and free bromine.

Physics Department,

F. C. ToY.

British Photographic Research Association. (Communication No. 66.)

\section{The Spacing of Young Trees.}

WiTH reference to the article on the spacing of young trees in NATURE of July 23, practical foresters in Great Britain might be glad to know the results of any experiments in spacing carried out in high and exposed situations and loose peaty soil. While the best results cannot be hoped for under these conditions, the comparative cheapness and unproductive character of land of this class render it tempting for afforestation with sitka spruce and perhaps other trees.

No. 3021, VoL. 120] 